\title{
Food Insecurity Is Associated with Prediabetes Risk Among U.S. Adolescents, NHANES 2003-2014
}

\author{
Arthur M. Lee, MD, ${ }^{1}$ Rebecca J. Scharf, MD, MPH, ${ }^{1}$ Stephanie L. Filipp, MPH, ${ }^{2}$ \\ Matthew J. Gurka, PhD, ${ }^{2}$ and Mark D. DeBoer, MD, MSc, MCR ${ }^{1}$
}

\begin{abstract}
Objective: Food insecurity has been linked to adverse health consequences. We sought to determine if food insecurity was related to obesity and prediabetes risk in U.S. adolescents. We also investigated if Supplemental Nutrition Assistance Program (SNAP) utilization mitigated any observed risks.

Materials and Methods: We used linear and logistic regression analysis of cross-sectional data from a nationally representative sample of U.S. adolescents aged 12-19 years participating in the National Health And Nutrition Examination Survey 2003-2014 who had an income:poverty ratio of $<5.0$ and had complete data regarding metabolic laboratory assessments, food security, and socioeconomic status $(n=2662)$.

Results: Food insecurity was present in $18.40 \%$ (95\% confidence interval (CI): 16.47-20.37) among U.S. adolescents meeting inclusion criteria. Food insecurity was associated with increased odds of elevated blood pressure (adjusted odds ratio $[\mathrm{aOR}]=1.57,95 \% \mathrm{CI}: 1.11-2.22)$ and prediabetes $(\mathrm{aOR}=1.94,95 \% \mathrm{CI}: 1.16-3.25)$. SNAP usage was associated with higher body mass index $z$-score (e.g., mean: $0.80 \mathrm{vs.} 0.59, P=0.02)$, increased insulin resistance, and increased prediabetes odds among food-secure adolescents relative to nonusage.

Conclusions: Food insecurity is relatively prevalent among U.S. adolescents with an income:poverty ratio of $<5.0$. Food insecurity is related to laboratory abnormalities and corresponding adverse health outcomes among U.S. adolescents in this relatively large sample. SNAP usage was associated with adverse health observations among food-secure adolescents. Ongoing efforts are still needed toward food assistance as important public health efforts aimed at mitigating the adverse outcomes related to food insecurity.
\end{abstract}

Keywords: food insecurity, adolescents, prediabetes, metabolic syndrome, obesity

\section{Introduction}

$\mathrm{F}$ OOD INSECURITY HAS been studied over several decades with data associating it with worse health outcomes, including poorer general health, poorer cognitive and developmental outcomes, obesity, and increased risk of hospitalization. $^{1-5}$ Despite food insecurity repeatedly being linked to adverse health outcomes, specific associations have been difficult to replicate across different studies and sample groups. Variations have been reported based on sex, age, socioeconomic status, geography, and other factors. ${ }^{6-9}$ Among children and adolescents, food insecurity has been linked both to being underweight (largely in developing areas of the world $)^{10-12}$ and to overweight or obesity (in developed areas), ${ }^{7,13,14}$ though the majority of these studies have been performed among younger children, leaving these relationships unclear among U.S. adolescents. Also unclear is whether these relationships with obesity extend to other metabolic consequences, including findings related to the metabolic syndrome (MetS).

It is important to understand how food insecurity plays a role in the current obesity epidemic among children and adolescents. While the prevalence of obesity may have plateaued, body mass index (BMI) $z$-scores have consistently trended upward in U.S. adolescents. ${ }^{15}$ Childhood and adolescent obesity portends other adverse outcomes such as MetS, prediabetes, cardiovascular disease, fatty liver disease, and renal dysfunction. ${ }^{16-19}$ Food insecurity is a potentially modifiable factor in the pathogenesis of the obesity epidemic. One potential means of mitigating food insecurity

\footnotetext{
${ }^{1}$ Department of Pediatrics, University of Virginia School of Medicine, Charlottesville, Virginia.

${ }^{2}$ Department of Health Outcomes \& Biomedical Informatics, University of Florida College of Medicine, Gainesville, Florida.
} 
is through the Supplemental Nutrition Assistance Program (SNAP), though data assessing food insecurity and SNAP use among adolescents are scarce.

In assessing these relationships among U.S. adolescents, we hypothesized the following: (1) food insecurity would be independently associated with obesity in U.S. adolescents, as we had previously demonstrated in U.S. kindergarten$\mathrm{ers}^{14}$; (2) among adolescents with available fasting laboratory data, food insecurity would be associated with metabolic abnormalities; and (3) SNAP utilization would mitigate the adverse associations with food insecurity, as had been demonstrated in previous studies. ${ }^{3,20}$

\section{Materials and Methods}

We examined participant data from the CDC NHANES from 2003 to 2014. NHANES is a cross-sectional, national, stratified, multistage probability survey conducted in 2-year waves with randomly selected noninstitutionalized U.S. civilians. We included multiple waves of the study to allow for improved power. Race/ethnicity was self-reported as white or black Hispanic, non-Hispanic white, or non-Hispanic black (subsequently referred to as Hispanic, white, and black, respectively). Racial/ethnic minorities and individuals $\leq 130 \%$ of the federal poverty level were intentionally oversampled. Sample weights were provided by NHANES to account for the oversampling as well as different response rates in an effort to create a nationally representative sample. ${ }^{21}$ Additional weights were created based on fasting status for laboratory measures. This study was approved by the National Center for Health Statistics ethics review board (Protocol Nos. 98-12, 2005-06, and 2011-17). Participants or their legal guardians provided informed consent.

Food security was assessed with the U.S. Department of Agriculture (USDA) 18-item household food security questionnaire. ${ }^{22,23}$ Ten questions were directed at the adult and household experience with food insecurity, and eight questions were directed at child-specific experiences. A composite scaled score and categorical classification of household food security was created using all 18 items, with positive responses to any of the questions indicating the presence of food insecurity. ${ }^{22,23}$ The food security questionnaire was administered only to participants with a household incometo-poverty ratio $<5.0$. SNAP utilization was self-reported and was asked of all participants (including those who were ineligible to receive SNAP benefits). There was an adjustment in survey questionnaire phrasing between 2006 and 2007, changing from if a household was "authorized" for SNAP to if a household "had received" SNAP benefits.

Dietary intake was determined based on two 24-hour food recall interviews administered by a trained dietary interviewer using a four-step multipass approach. The first recall interview was administered on examination day at the mobile examination centers. The second recall interview was administered by telephone. Interview data were processed and coded to determine specific macronutrient intake based on the USDA National Nutrient Database for Standard Reference. ${ }^{24,25}$ Total macronutrient intake (in grams) was calculated as an average of the two 24-hour food recall interviews. Macronutrient consumption was reported as percentage of total calories accounted by specific food group. ${ }^{26}$ The equations were as follows: \% total energy fromcarbohydrates $(4 \times$ grams of carbohydrates)/total calories, protein $(4 \times$ grams of protein $) /$ total calories, fats $(9 \times$ grams of fats)/total calories. Physical activity data did not have a consistently surveyed variable until the 2007-2008 wave. Starting then, physical activity was self-reported as average daily minutes of moderate-to-vigorous physical activity.

Clinical and fasting laboratory measurements were obtained each year using controlled equipment and protocols. Fasting samples were obtained from participants who attended morning sessions at the mobile examination centers. $z$-scores were calculated according to the U.S. CDC 2000 Growth Reference adjusting for age and gender. ${ }^{27}$ Elevated blood pressure (BP) was defined as average systolic or diastolic BP exceeding the 90th percentile for age, sex, and height, or being on antihypertensive medication. ${ }^{28}$ MetS was evaluated with the adolescent adaptation of the Adult Treatment Panel III (ATP-III) criteria and with the MetS severity score (MetS $z$-score-http://mets.health-outcomespolicy.ufl.edu/calculator). ATP-III MetS was defined as having at least three of five: elevated BP, BMI $z$-score, fasting glucose, fasting triglycerides, or low high-density lipoprotein (HDL). ${ }^{29-3}$ Prediabetes was defined per criteria from the American Diabetes Association with glycosylated hemoglobin (HbA1C) between 5.7\%-6.4\%. ${ }^{32}$ Because of small numbers of those with diabetes, we combined prediabetes and diabetes for analysis.

Participants aged 12-19 were included for analysis. Only Hispanic, white, and black participants were included because the MetS $z$-score has not been derived for other race/ethnicity groups. Participants were excluded on the basis of following factors: antidiabetic medication usage, pregnant at the time of examination, nonfasting status, incomplete laboratory data regarding MetS, incomplete household income data, incomplete parental education data, and incomplete food security assessment. The final sample only included adolescents with household income-topoverty-ratio $<4.0$ due to the food security assessment administration. Antidiabetic medication usage and pregnancy status were used as exclusion criteria, given their potential to alter laboratory data and/or current weight status. It is important to note for the major excluding factor (incomplete laboratory data), fasting laboratory data were only drawn on half of all NHANES participants, those who are attending morning sessions at the mobile examination centers.

Statistical analyses were performed with SAS (version 9.4, Cary, NC). Survey procedures were used to account for the survey design and incorporated sample weights as provided by NHANES. Least-squares mean analyses were used to show differences in clinical measurements between different groups. Logistic regression analyses were used to show differences in clinical outcomes between different groups. Out of concern for the potential for confounding, all statistical analyses adjusted for age, sex, race/ethnicity, and household income-to-poverty ratio, as performed in previous NHANES assessments of food insecurity in other age ranges. ${ }^{13,20}$ For all logistic regression models, we assessed for possible interactions that food insecurity might have with age, sex, and income (separately). Having found not such interactions, the interaction terms were dropped from the models. We assessed collinearity between income and insecurity through the variance inflation factor; values were $<2$, indicating no collinearity. Because of the large number of participants being excluded due to nonfasting status, we performed a sensitivity analysis evaluating all participants 
with data regarding food insecurity status and family income for relationships with body weight status, BP, and $\mathrm{HbA1C}$, which does not require fasting status.

\section{Results}

We analyzed data from 2662 participants with complete data as previously described. Table 1 shows the demographic data of the sample. Food insecurity was present in $18.40 \%$ (95\% confidence interval [CI]: 16.47-20.37) of the sample. Chi-square tests revealed significant differences in the distribution of food insecurity within groups by race/ethnicity and income-to-poverty ratio. In direct comparison with white participants, the proportion of the food-insecure participants was higher in Hispanic and black adolescents (both $P<0.0001)$. The proportion of food-insecure participants was higher in those with lower household income-to-poverty ratio $<1.0$ than for $2.0-3.0(P<0.0001)$ and in those whose parental education ended at high school compared with those beyond high school $(P<0.0001)$. There was no statistically significant difference in food insecurity prevalence based on sex in our sample. Excluded participant characteristics are shown in Supplementary Table S1. There were no differences based on sex, household income, or parental education. There was a difference within race/ethnicity due to all participants in the "other" race/ethnicity category (Asian, Pacific Islander, Native American/Alaskan, and mixed) being excluded because the MetS $z$-score has not been derived for these groups yet.

Of the final sample, $3.64 \%$ (95\% CI: 2.72-4.56) had prediabetes as determined by $\mathrm{HbA} 1 \mathrm{C}$, whereas only $0.12 \%$ (95\% CI: 0.00-0.25) had diabetes. In subsequent analyses, the small percentage of participants with diabetes was grouped with the participants with prediabetes.

Table 2 shows metabolic differences based on food insecurity status. Food-insecure adolescents had significantly higher $\operatorname{HbA1C}(5.21$ vs. $5.16, P=0.03)$ and increased odds of elevated $\mathrm{BP}$ (adjusted odds ratio [aOR] $=1.65,95 \% \mathrm{CI}: 1.38-1.98)$ and prediabetes/diabetes $(\mathrm{aOR}=1.96,95 \% \mathrm{CI}$ : 1.17-3.19). Notably, food-insecure adolescents overall did not have a higher BMI $z$-score, or increased odds of ATP-III MetS, being either overweight or obese, or being obese. We did note potential trends toward higher BMI $z$-score $(P=0.06)$ and higher MetS $z$ score $(P=0.11)$ in the food-insecure group.

Table 3 shows differences in macronutrient consumption based on food insecurity status and SNAP utilization. Not all participants in the sample had complete data regarding dietary intake and SNAP. In a subset of 2392 participants with complete data regarding dietary intake and food insecurity, food-insecure adolescents ate fewer total calories than their

Table 1. Participant Demographics ${ }^{\mathrm{a}}$

\begin{tabular}{|c|c|c|c|c|c|c|}
\hline & \multicolumn{2}{|r|}{ Total sample } & \multicolumn{2}{|r|}{ Food secure } & \multicolumn{2}{|r|}{ Food insecure } \\
\hline & $\mathrm{n}$ & $\%(95 \% C I)$ & $\mathrm{n}$ & $\%(95 \% C I)$ & $\mathrm{n}$ & $\%(95 \% C I)$ \\
\hline & 2662 & & 1977 & $81.58(79.63-83.53)$ & 685 & $18.40(16.47-20.37)$ \\
\hline \multicolumn{7}{|c|}{ 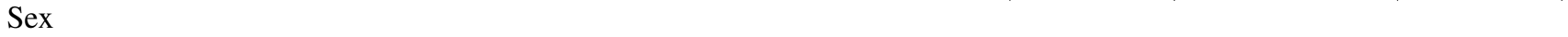 } \\
\hline Female & 1225 & $48.14(45.17-51.11)$ & 915 & 82.49 (80.02-94.97) & 310 & $17.51(15.03-19.98)$ \\
\hline \multirow[t]{2}{*}{ Male } & 1437 & $51.86(48.89-54.83)$ & 1062 & $80.73(78.06-83.40)$ & 375 & $19.27(16.60-21.94)$ \\
\hline & & & & & & $P=0.30$ \\
\hline \multicolumn{7}{|l|}{ Race ethnicity } \\
\hline Hispanic & 986 & $18.76(15.49-22.03)$ & 680 & $70.53(66.60-74.45)$ & 306 & $29.47(25.55-33.40)$ \\
\hline White & 819 & $66.08(61.87-70.29)$ & 683 & 87.37 (85.17-89.57) & 136 & $12.63(10.43-14.83)$ \\
\hline \multirow[t]{2}{*}{ Black } & 857 & $15.16(12.66-17.66)$ & 614 & $70.00(65.39-74.62)$ & 243 & $30.00(25.38-34.61)$ \\
\hline & & & & & & $P<0.0001$ \\
\hline \multicolumn{7}{|l|}{ Income:poverty } \\
\hline $4.0-5.0$ & 444 & $27.35(23.81-30.89)$ & 437 & $98.61(85.93-100.00)$ & 7 & $1.39(0.15-2.62)$ \\
\hline $3.0-4.0$ & 279 & $14.25(11.44-17.05)$ & 254 & $91.86(72.62-100.00)$ & 25 & $8.13(3.29-100.00)$ \\
\hline $2.0-3.0$ & 358 & $14.41(12.36-16.36)$ & 290 & $84.15(69.60-98.69)$ & 68 & $15.85(10.06-21.64)$ \\
\hline $1.0-2.0$ & 715 & $21.73(19.47-24.00)$ & 493 & $71.30(61.63-80.97)$ & 222 & $28.70(24.06-33.33)$ \\
\hline \multirow[t]{2}{*}{$<1.0$} & 866 & $22.26(19.26-25.26)$ & 503 & $62.44(56.82-68.06)$ & 363 & $37.56(31.94-43.18)$ \\
\hline & & & & & & $P<0.0001$ \\
\hline \multicolumn{7}{|l|}{ Parental education } \\
\hline No HS diploma & 702 & $15.09(13.02-17.16)$ & 436 & $64.68(59.87-69.50)$ & 266 & $35.32(30.50-40.13)$ \\
\hline HS diploma & 570 & $19.71(16.83-22.50)$ & 389 & $74.76(69.27-80.25)$ & 181 & $25.24(19.75-30.73)$ \\
\hline \multirow[t]{2}{*}{$>$ HS diploma } & 1390 & $65.20(61.59-68.80)$ & 1152 & $87.55(85.49-89.62)$ & 238 & $12.45(10.38-14.51)$ \\
\hline & & & & & & $P<0.0001$ \\
\hline SNAP use (subset of sample) & 1885 & & 1346 & & 539 & \\
\hline Used SNAP & 626 & $33.21(28.01-38.41)$ & 341 & $25.33(21.35-29.32)$ & 285 & $52.88(42.96-62.80)$ \\
\hline \multirow[t]{2}{*}{ Did not use SNAP } & 1259 & $66.79(60.78-72.80)$ & 1005 & 74.67 (69.74-79.59) & 254 & $47.12(38.44-55.81)$ \\
\hline & & & & & & $P<0.0001$ \\
\hline
\end{tabular}

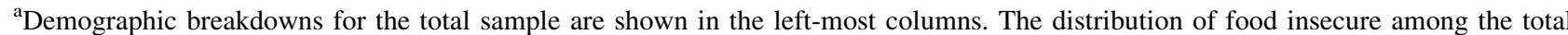
sample is shown in the center and right columns. Unweighted $n$ 's and weighted percentages with $95 \%$ CIs are reported. Statistical significance was determined through chi-square testing for difference in prevalence between groups by food security status and by demographic characteristic subcategory.

${ }^{\mathrm{b}}$ Food security was assessed with the U.S. Department of Agriculture (USDA) 18-item household food security questionnaire, ${ }^{22}$ with positive response to any of the questions indicating the presence of food insecurity.

CI, confidence interval; SNAP, Supplemental Nutrition Assistance Program.
} 
Table 2. Clinical Measurements and Outcomes Based on Food Security Status ${ }^{\mathrm{a}}$

\begin{tabular}{|c|c|c|c|c|}
\hline & Overall & Food secure & Food insecure & \\
\hline $\mathrm{N}$ & 2662 & 1977 & 685 & food secure \\
\hline Clinical outcomes & $\%(95 \% C I)$ & $\%(95 \% C I)$ & $\%(95 \% C I)$ & aOR $(95 \% C I)$ \\
\hline ATP-III MetS ${ }^{b}$ & $7.27(5.83-8.71)$ & $6.58(5.05-8.11)$ & $10.30(7.33-13.27)$ & $1.23(0.80-1.90)$ \\
\hline Overweight or obese ${ }^{c}$ & $36.56(34.07-39.04)$ & $35.62(32.72-38.53)$ & $40.68(35.89-45.48)$ & $1.14(0.85-1.52)$ \\
\hline Obese $^{c}$ & $20.23(17.92-22.54)$ & $19.52(16.93-22.10)$ & $23.37(19.16-27.58)$ & $1.08(0.83-1.41)$ \\
\hline Elevated $\mathrm{BP}^{\mathrm{d}}$ & $7.43(5.85-9.02)$ & $6.50(4.88-8.13)$ & $11.55(8.62-14.48)$ & $1.65(1.38-1.98)$ \\
\hline Prediabetes or diabetes ${ }^{\mathrm{e}}$ & $3.65(2.72-4.58)$ & $2.89(1.98-3.81)$ & 6.98 (4.41-9.55) & $1.96(1.17-3.19)$ \\
\hline Clinical measurements & Mean $(95 \%$ CI) & Mean $(95 \%$ CI) & Mean $(95 \%$ CI) & $\mathrm{P}$ \\
\hline MetS z-score & $-0.03(-0.07-0.01)$ & $-0.02(-0.06-0.03)$ & $0.06(-0.02-0.13)$ & 0.11 \\
\hline BMI z-score & $0.65(0.58-0.69)$ & $0.64(0.57-0.70)$ & $0.76(0.65-0.87)$ & 0.06 \\
\hline HDL (mg/dL) & $52.63(52.02-53.25)$ & $52.28(51.63-52.93)$ & $52.47(51.27-53.67)$ & 0.78 \\
\hline Triglycerides (mg/dL) & $85.72(82.73-88.71)$ & $86.32(83.20-89.44)$ & $89.98(84.28-95.67)$ & 0.22 \\
\hline Glucose $(\mathrm{mg} / \mathrm{dL})$ & $93.04(92.50-93.57)$ & 93.37 (92.77-93.97) & $93.11(92.13-94.09)$ & 0.64 \\
\hline Insulin $(\mu \mathrm{U} / \mathrm{mL})$ & $12.16(11.65-12.68)$ & $12.46(11.83-13.10)$ & $13.52(12.25-14.78)$ & 0.13 \\
\hline $\mathrm{HbA} 1 \mathrm{C}(\%)$ & $5.17(5.15-5.19)$ & $5.16(5.14-5.18)$ & $5.21(5.17-5.24)$ & $\mathbf{0 . 0 3}$ \\
\hline
\end{tabular}

Bold text refers to statistically significant differences between the food secure and food insecure groups $(P<0.05)$.

${ }^{a}$ Unweighted $n$ 's and weighted percentages/means with 95\% CIs are reported. ORs (comparing clinical outcomes between those food secure and insecure) and mean levels are all reported following adjustment of age, sex, race/ethnicity, and household income-to-poverty ratio. All clinical outcomes and clinical measurements were modeled separately.

${ }^{\mathrm{b}}$ At least three of the five following: elevated BMI percentile $\geq 85$ th\%, BP, fasting triglycerides, fasting glucose, or low HDL.

${ }^{\mathrm{c}}$ Overweight defined as BMI percentile 85 to $<95$; obese defined as BMI percentile $\geq 95$ th.

${ }^{\mathrm{d}}$ Defined as $>90$ th percentile for age, sex, and height.

${ }^{\mathrm{e}}$ Prediabetes defined as A1C $5.7 \%-6.4 \%$, diabetes defined as $\mathrm{A} 1 \mathrm{C}>6.4 \%$.

aOR, adjusted odds ratio; ATP, Adult Treatment Panel; BMI, body mass index; BP, blood pressure; HbA1C, glycosylated hemoglobin; HDL, high-density lipoprotein; MetS, metabolic syndrome.

food-secure counterparts (1996 vs. 2138, $P=0.03$ ). There were no differences in macronutrient consumption, though there was a potential trend toward lower percentage of saturated fat intake in those who were food insecure $(P=0.08)$. In a subset of 1704 participants with complete data regarding dietary intake and SNAP usage, total calories and macronutrient consumption did not significantly differ based on food stamps usage.

In addition, there were no differences in self-reported physical activity based on food insecurity status regardless of sex or household income in the sample of participants from 2007 to 2014 ( $n=894)$ whom these data were available for.

Table 3. Macronutrient Consumption Differences Based on Food Security Status and SNaP Usage ${ }^{a}$

\begin{tabular}{|c|c|c|c|c|}
\hline \multirow[b]{3}{*}{ Food security status } & Overall & Food secure & Food insecure & \multirow{2}{*}{$\begin{array}{c}\text { Food insecure } \\
\text { vs. secure }\end{array}$} \\
\hline & $\mathrm{n}=2392$ & $\mathrm{n}=1795$ & $\mathrm{n}=597$ & \\
\hline & Mean $(95 \%$ CI) & Mean $(95 \%$ CI) & Mean $(95 \%$ CI) & $\mathrm{P}$ \\
\hline Calories & $2114(2065-2163)$ & 2138 (2079-2196) & 1996 (1896-2095) & 0.03 \\
\hline$\%$ carbohydrates & $52.74(52.18-53.30)$ & $52.61(52.03-53.20)$ & $53.32(52.02-54.61)$ & 0.32 \\
\hline$\%$ protein & $14.94(14.71-15.12)$ & $14.90(14.69-14.37)$ & $14.84(14.37-15.30)$ & 0.77 \\
\hline$\%$ saturated fat & $11.27(11.11-11.45)$ & $11.34(11.14-11.54)$ & $10.96(10.59-11.32)$ & 0.08 \\
\hline \multirow[t]{3}{*}{$\%$ total fat } & $33.05(32.59-33.51)$ & $33.18(32.62-33.73)$ & $32.57(31.58-33.55)$ & 0.31 \\
\hline & Overall & Did not use SNAP & Used SNAP & \multirow{2}{*}{$\begin{array}{l}\text { SNAP usage } \\
\text { vs. nonusage }\end{array}$} \\
\hline & $\mathrm{n}=1704$ & $\mathrm{n}=1165$ & $\mathrm{n}=539$ & \\
\hline SNAP utilization & Mean $(95 \%$ CI $)$ & Mean $(95 \%$ CI) & Mean $(95 \%$ CI $)$ & $\mathrm{P}$ \\
\hline Calories & $2154(2098-2209)$ & $2169(2148-2190)$ & $2061(1706-2416)$ & 0.34 \\
\hline$\%$ carbohydrates & $53.01(52.29-53.73)$ & $52.55(48.83-56.28)$ & $54.12(49.03-59.22)$ & 0.52 \\
\hline$\%$ protein & $14.62(14.40-14.85)$ & $14.71(14.03-15.40)$ & $14.34(13.14-15.54)$ & 0.48 \\
\hline$\%$ saturated fat & $11.30(11.07-11.53)$ & $11.48(10.20-12.75)$ & $10.83(8.70-12.97)$ & 0.50 \\
\hline$\%$ total fat & $33.00(32.38-33.62)$ & $33.25(30.20-36.30)$ & $32.29(27.81-36.77)$ & 0.64 \\
\hline
\end{tabular}

Bold text refers to statistically significant differences between the food secure and food insecure groups $(P<0.05)$.

${ }^{a}$ Food recall and food stamps usage data were not complete for the entire sample. Unweighted $n$ 's and weighted statistics are reported. All analyses controlled for sex, age, race/ethnicity, and household income. 


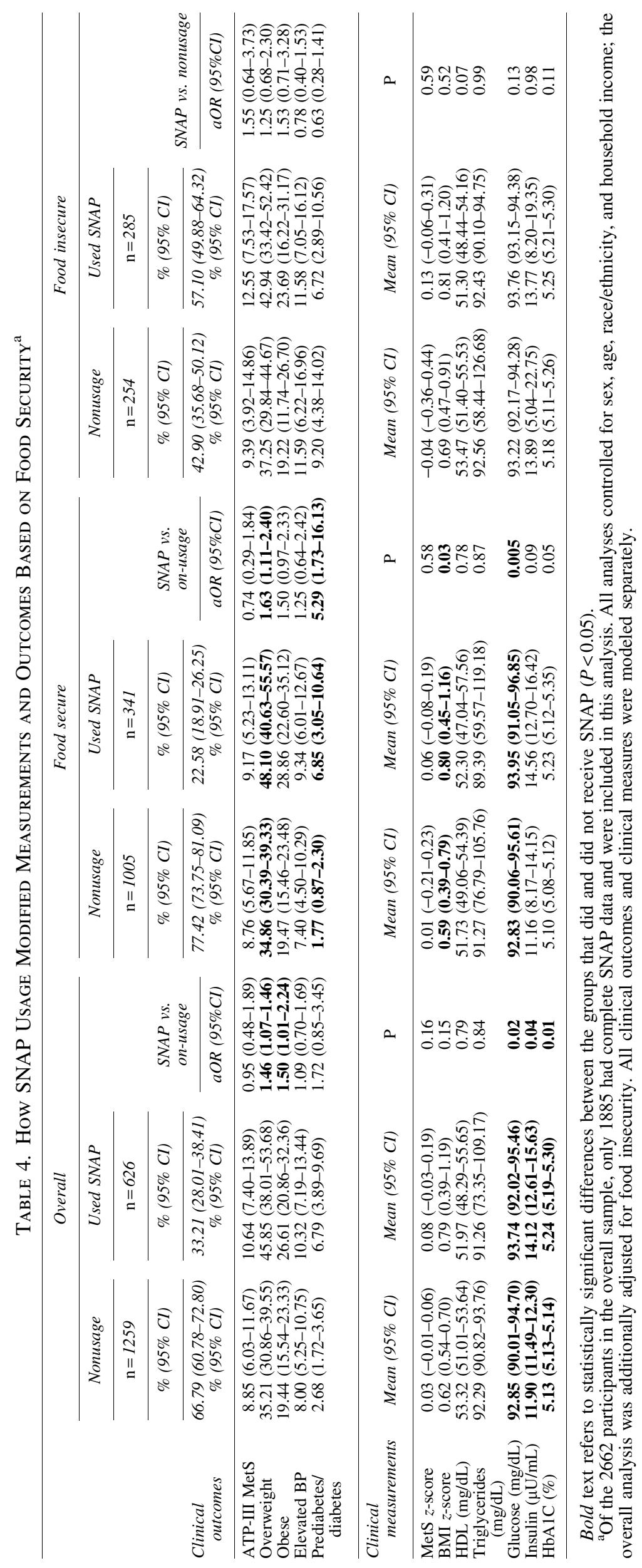




\section{SNAP utilization among food-secure adolescents}

Table 4 shows how SNAP utilization affected clinical measurements and outcomes within food-secure and insecure participants. Food-secure adolescents who used SNAP (compared with nonusage) had higher BMI $z$-score (0.80 vs. $0.59, P=0.03)$ and fasting glucose measurements (93.95 vs. $92.83, P=0.005)$. This corresponded with significantly increased overweight/obese $(\mathrm{aOR}=1.63,95 \% \mathrm{CI}: 1.11-2.40)$ and prediabetes/diabetes $(\mathrm{aOR}=5.29,95 \% \mathrm{CI}: 1.73-16.13)$ odds. There was also a potential trend toward higher $\mathrm{HbA} 1 \mathrm{C}$ among SNAP users vs. nonusers $(P=0.05)$.

\section{SNAP utilization among food-insecure adolescents}

Table 4 shows how SNAP usage affected clinical measurements and outcomes, both overall (adjusted for food insecurity) and separately within food-secure and insecure participants. In the overall sample, SNAP use was associated with higher odds of overweight and obesity, as well as higher levels of glucose, insulin, and HbA1C. Food-insecure adolescents who used SNAP (compared with nonusage) showed no differences in any of the anthropometric or laboratory measurements. SNAP usage in this group showed no difference in odds for ATP-III MetS, overweight status, elevated $\mathrm{BP}$, or prediabetes/diabetes. There was also a potential trend toward lower HDL cholesterol $(P=0.07)$.

\section{Sensitivity analysis}

Given that we excluded a high number of participants on the basis of incomplete fasting laboratory data for MetS evaluation, we performed a sensitivity analysis among participants with complete food security assessment and household income data (no other excluding criteria). Our goal was to examine whether the observed associations of food insecurity with increased elevated BP and prediabetes/diabetes odds, and the lack of association with BMI $z$-score and overweight/obesity odds were reproducible outside of the small restricted sample used for our primary analysis.

There were 8175 adolescents included in this sensitivity analysis sample. The multivariate model adjusted for age, sex, and household income consistent with our previous models. We performed the analyses with nonfasting weights, given that complete fasting laboratory data were no longer an inclusion criterion. We found that food-insecure adolescents had no significant differences in BMI $z$-score (difference estimate $=-0.07$, 95\% CI: $-0.16-0.01, P=0.10)$. Food-insecure adolescents had elevated odds of high $\mathrm{BP}(\mathrm{aOR}=1.35,95 \% \mathrm{CI}$ : $1.08-1.69)$.

Among these 8175 participants, 8061 had HbA1C (nonfasting laboratory) data for which we also analyzed prediabetes/diabetes outcomes. Food-insecure adolescents in this sensitivity analysis had significantly increased HbA1C (5.23 vs. $5.18, P=0.006)$ and increased odds of prediabetes/diabetes $(\mathrm{aOR}=1.59,95 \% \mathrm{CI}: 1.22-2.07)$. These findings are all consistent with our central analysis.

\section{Discussion}

Our study contributes to the extensive field of food insecurity by showing associations of abnormal laboratory measurements and corresponding clinical outcomes with food insecurity among U.S. adolescents. In this cohort of
2662 U.S. adolescents, food-insecure adolescents were more likely to have elevated $\mathrm{HbA} 1 \mathrm{C}$, and had higher odds of elevated BP and prediabetes/diabetes. Contrary to our expectations, we found that while there appeared to be trends toward higher BMI $z$-scores among those who were food insecure, overall food insecurity was not independently associated with obesity within this sample. In a sensitivity analysis with a larger sample, we confirmed the observations of the central analysis. This is important because our final sample excluded a large number of participants on the basis of incomplete fasting laboratory data. This sensitivity analysis showed that these associations can be observed (or, in the case of obesity, observed to be not significantly associated) across a larger sample, and that our final sample was adequately powered to detect them.

How food insecurity is mechanistically related to weight status and prediabetes is unclear. One possible theory is that food insecurity fosters unhealthy eating behaviors in children and adolescents, translating to poor health markers after prolonged establishment of these habits. ${ }^{33}$ Another theory is that food-insecure children are more dependent on less nutritious and more obesogenic foods, perpetuating the negative outcomes. ${ }^{34,35}$ A third theory is that food insecurity is more of a marker of poorer socioeconomic status and does not independently influence health outcomes. ${ }^{8,36}$ Our data did not show any differences in macronutrient consumption based on food security status, with only a potential trend of lower saturated fat in the food-insecure group $(P=0.08)$, which was in the opposite direction of what one might have guessed for higher metabolic risk. However, macronutrient analysis is a superficial analysis. For example, percentage carbohydrate consumption will not differentiate between the complex and simple sugars that may affect a food's glycemic index. Based on our data, we could not support any specific theory linking food insecurity with weight status and prediabetes. Additionally related to lifestyle factors, there were no significant differences in self-reported exercise based on food security status.

Addressing food insecurity through food assistance programs has the potential to reduce development of adverse health outcomes in children. Previous studies have shown that SNAP positively influenced health among food-insecure children. ${ }^{3,20}$ However, our data showed adverse health outcomes associated with SNAP utilization among food-secure adolescents. SNAP usage among food-secure U.S. adolescents was related to significantly increased odds of being overweight/obese and having prediabetes/diabetes. This was correlated with increased BMI $z$-score and fasting glucose in that group, and with a trend toward lower HDL $(P=0.07)$. Although the statistically significant difference in fasting glucose in that group appears clinically insignificant, it was associated with the increased odds of prediabetes/diabetes.

The association of SNAP utilization and increased weight status and prediabetes odds could have multiple explanations. One theory previously reported is that families dependent on SNAP are more likely to develop a feast-famine cycle, characterized by 3 weeks of abundant food consumption and 1 week of relative food insufficiency. $6,37,38$ The existence of this cycle is further supported by reports of families consistently running out of their SNAP benefits allotment before the month's end. ${ }^{38}$ This feast-famine cycle fosters the development of binge-eating behaviors that result in weight gain over time. ${ }^{6,37}$ Insulin resistance may 
subsequently develop in conjunction with weight gain and abnormal eating patterns. ${ }^{37,39}$ We were limited by the nature of the NHANES data, and we could not make any conclusions explaining the observed association of SNAP usage with increased prediabetes/diabetes and overweight/obese odds in food-secure adolescents.

We want to emphasize that our study does not identify SNAP as a driver of adverse health associations in U.S. adolescents. Other studies have shown that SNAP is associated with significant health benefits among food-insecure children. $^{3,20}$ Our data showing SNAP associated with increased odds of prediabetes/diabetes and overweight/obesity in foodsecure children suggest that there exists room for improvement within SNAP. Policy-based changes can be considered to improve the system. ${ }^{6,40}$ If the current monthly distribution plan propagates a feast-famine cycle, ${ }^{37-40}$ a 2 -week distribution cycle may reduce this risk. Increasing nutrition and budget management education among SNAP recipients could also mitigate adverse outcomes associated with the feastfamine cycle. ${ }^{41}$ Improving access to healthier nutritious foods would provide potential benefit to SNAP recipients. ${ }^{41}$

There are alternative theories on why SNAP utilization has been associated with overweight/obesity and prediabetes/diabetes odds. Households who receive SNAP benefits could differ from those who elect not to. These households could be more likely to have experienced hunger, which is a more severe variant of food insecurity. They may have experienced a greater duration of food insecurity, with duration being the driving factor of abnormal eating behaviors rather than the feastfamine cycle. ${ }^{42}$ NHANES is limited in its ability to assess food insecurity duration due to the cross-sectional design.

Our study had several important limitations, especially given the survey design of NHANES. Due to the crosssectional nature, we are only able to report correlations and precise causation cannot be ascertained. Another limitation is that physical activity was not consistently assessed 20032014 and was dependent on self-reporting. Per our analyses, the sample mean minutes of daily exercise well exceeded current recommendations, yet the Youth Risk Behavior Surveillance showed that there is increasing sedentary behavior in U.S. adolescents. ${ }^{43}$ Our analytic sample was highly restricted, given the requirement for having complete data regarding laboratory, socioeconomic, and food security assessment. For example, food insecurity was designed to be assessed only among individuals with an income:poverty ratio $<5.0$ (which in the most recent year of NHANES analyzed [2014] represented an income of $\sim \$ 119,000$ for a household of 4). This limits the generalizability of our findings to the whole of the U.S. adolescent population, which is a common limitation in many studies examining food insecurity. In addition, a large number of participants did not answer questions regarding SNAP use, further limiting our conclusions on the intersection between SNAP use, food insecurity, and metabolic outcomes. We also excluded individuals on medicine, which might have affected MetS severity levels, though this applied to only a small number of adolescents $(n=19)$. However despite our high number of excluded participants, the final sample and excluded participants did not differ significantly in terms of food insecurity and socioeconomic status. Indeed, we had adequate power such that some of the differences between groups may not be seen as clinically significant, though they may still reveal important differences on a population basis.

\section{Conclusions}

Food insecurity has been repeatedly linked to adverse health outcomes across various subgroups of the United States. Our study shows that among adolescents, food insecurity most significantly carries increased odds of elevated $\mathrm{BP}$ and prediabetes. The primary intervention of prediabetes is based on lifestyle changes, which can prevent the continuation toward type 2 diabetes mellitus, ${ }^{44}$ a significant burden to both individuals and society. Thus, programs addressing food security such as SNAP are vital to public health efforts. Our findings associating use of SNAP with higher weight and prediabetes may highlight the need for further assessment of current practice regarding food security management in the United States.

\section{Author Disclosure Statement}

No competing financial interests exist.

\section{Funding Information}

This work was supported by the National Institutes of Health Grant 1R01HL120960. The NIH had no role in the design, analysis, or writing of this article.

\section{Supplementary Material}

\section{Supplementary Table S1}

\section{References}

1. Nord M. What have we learned from two decades of research on household food security? Public Health Nutr 2014;17:2-4.

2. Coleman-Jensen A, McFall W, Nord M. Food Insecurity in Households with Children: Prevalence, Severity, and Household Characteristics. Washington, DC: U.S. Department of Agriculture: Economic Research Service; 2013.

3. Cook JT, Frank DA, Berkowitz C, et al. Food insecurity is associated with adverse health outcomes among human infants and toddlers. $J$ Nutr 2004;134:1432-1438.

4. Ryu JH, Bartfeld JS. Household food insecurity during childhood and subsequent health status: The early childhood longitudinal study-kindergarten cohort. Am J Public Health 2012;102:e50-e55.

5. Johnson AD, Markowitz AJ. Associations between household food insecurity in early childhood and children's kindergarten skills. Child Dev 2017;89:e1-e17.

6. Dinour LM, Bergen D, Yeh MC. The food insecurityobesity paradox: A review of the literature and the role food stamps may play. J Am Diet Assoc 2007;107:1952-1961.

7. Rose D, Bodor JN. Household food insecurity and overweight status in young school children: Results from the Early Childhood Longitudinal Study. Pediatrics 2006;117:464-473.

8. Alaimo K, Olson CM, Frongillo EA, et al. Food insufficiency, family income, and health in US preschool and school-aged children. Am J Public Health 2001;91:781-786.

9. Kaur J, Lamb MM, Ogden CL. The Association between Food Insecurity and Obesity in Children-The National Health and Nutrition Examination Survey. J Acad Nutr Diet 2015; 115:751-758.

10. Ali D, Saha KK, Nguyen PH, et al. Household food insecurity is associated with higher child undernutrition in Bangladesh, Ethiopia, and Vietnam, but the effect is not mediated by child dietary diversity. J Nutr 2013;143:2015-2021. 
11. Tiwari R, Ausman LM, Agho KE. Determinants of stunting and severe stunting among under-fives: Evidence from the 2011 Nepal Demographic and Health Survey. BMC Pediatr 2014;14: 239.

12. Mutisya M, Kandala NB, Ngware MW, et al. Household food (in)security and nutritional status of urban poor children aged 6 to 23 months in Kenya. BMC Public Health 2015;15:1052.

13. Casey PH, Simpson PM, Gossett JM, et al. The association of child and household food insecurity with childhood overweight status. Pediatrics 2006;118:e1406-e1413.

14. Lee AM, Scharf RJ, DeBoer MD. Association between kindergarten and first-grade food insecurity and weight status in U.S. children. Nutrition 2018;51-52:1-5.

15. Lee AM, Gurka MJ, DeBoer MD. Trends in metabolic syndrome severity and lifestyle factors among adolescents. Pediatrics 2016;137:1-9.

16. Nichols GA, Hillier TA, Brown JB. Progression from newly acquired impaired fasting glusose to type 2 diabetes. Diabetes Care 2007;30:228-233.

17. Lee AM, Gurka MJ, DeBoer MD. Correlation of metabolic syndrome severity with cardiovascular health markers in adolescents. Metabolism 2017;69:87-95.

18. Fermin CR, Lee AM, Filipp SL, et al. Serum alanine aminotransferase trends and their relationship with obesity and metabolic syndrome in United States adolescents, 1999-2014. Metab Syndr Relat Disord 2017;15:276-282.

19. Henegar JR, Bigler SA, Henegar LK, et al. Functional and structural changes in the kidney in the early stages of obesity. J Am Soc Nephrol 2001;12:1211-1217.

20. Nguyen BT, Ford CN, Yaroch AL, et al. Food security and weight status in children: interactions with food assistance programs. Am J Prev Med 2017;52:S138-S144.

21. National_Center_for_Health_Statistics C. National Health and Nutrition Examination Survey Protocol, 2016. Accessed at www.cdc.gov/nchs/nhanes/nhanes_questionnaires .htm on December 7.

22. Guide to Measuring Household Food Security. Alexandria, VA: U.S. Department of Agriculture: Food and Nutrition Service; 2000.

23. Measuring Children's Food Security in U.S. Households, 1995-99. Washington, DC: U.S. Department of Agriculture: Economic Research Service; 2002.

24. USDA. National Nutrient Database for Standard Reference. Accessed at http://ars.usda.gov/ba/bhncr/ndl on August 14, 2015

25. Lee AM, Scharf RJ, DeBoer MD. Food insecurity is associated with prediabetes and dietary differences in U.S adults aged 20-39. Prev Med 2018;116:180-185.

26. Reedy J, Krebs-Smith SM. Dietary sources of energy, solid fats, and added sugars among children and adolescents in the United States. J Am Diet Assoc 2010;110:1477-1484.

27. Kuczmarski RJ, Ogden CL, Grummer-Strawn LM, et al. CDC growth charts: United States. Adv Data 2000;314: $1-27$.

28. Kaplan N. Kaplan's Clinical Hypertension. Philadelphia, PA: Lippincott Williams \& Wilkins; 2010.

29. Gurka MJ, Ice CL, Sun SS, et al. A confirmatory factor analysis of the metabolic syndrome in adolescents: An examination of sex and racial/ethnic differences. Cardiovasc Diabetol 2012;11:128.

30. Grundy SM, Cleeman JI, Daniels SR, et al. Diagnosis and management of the metabolic syndrome: An American
Heart Association/National Heart, Lung, and Blood Institute Scientific Statement. Circulation 2005;112:27352752.

31. Alberti KG, Eckel RH, Grundy SM, et al. Harmonizing the metabolic syndrome: A joint interim statement of the International Diabetes Federation Task Force on Epidemiology and Prevention; National Heart, Lung, and Blood Institute; American Heart Association; World Heart Federation; International Atherosclerosis Society; and International Association for the Study of Obesity. Circulation 2009;120:1640-1645.

32. Amer Diabet A. Standards of medical care in diabetes2014. Diabetes Care 2014;37:S14-S80.

33. Fisher JO, Birch LL. Restricting access to palatable foods affects children's behavioral response, food selection, and intake. Am J Clin Nutr 1999;69:1264-1272.

34. Drewnowski A. Fat and sugar: An economic analysis. J Nutr 2003;133:838S-840S.

35. Drewnowski A, Rehm CD. Consumption of added sugars among US children and adults by food purchase location and food source. Am J Clin Nutr 2014;100:901-907.

36. Laraia BA, Siega-Riz AM, Evenson KR. Self-reported overweight and obesity are not associated with concern about enough food among adults in New York and Louisiana. Prev Med 2004;38:175-181.

37. Townsend MS, Peerson J, Love B, et al. Food insecurity is positively related to overweight in women. J Nutr 2001; 131:1738-1745.

38. Taren DL, Clark W, Chernesky M, et al. Weekly food servings and participation in social programs among low income families. Am J Public Health 1990;80:1376-1378.

39. Hellström PM, Geliebter A, Näslund E, et al. Peripheral and central signals in the control of eating in normal, obese and binge-eating human subjects. Br J Nutr 2004;92:S47S57.

40. Wilde PE, Ranney CK. The monthly food stamp cycle: Shopping frequency and food intake decisions in an endogenous switching regression framework. Am J Agric Econ 2000;82:200-213.

41. Wiig K, Smith C. The art of grocery shopping on a food stamp budget: Factors influencing the food choices of lowincome women as they try to make ends meet. Public Health Nutr 2009;12:1726-1734.

42. Wilde PE. Measuring the effect of food stamps on food insecurity and hunger: Research and policy considerations. J Nutr 2007;137:307-310.

43. Eisenmann JC, Bartee RT, Wang MQ. Physical activity, TV viewing, and weight in U.S. youth: 1999 Youth Risk Behavior Survey. Obes Res 2002;10:379-385.

44. Knowler WC, Barrett-Connor E, Fowler SE, et al. Reduction in the incidence of type 2 diabetes with lifestyle intervention or metformin. N Engl J Med 2002;346:393-403.

Address correspondence to: Mark D. DeBoer, $M D, M S c, M C R$ Department of Pediatrics University of Virginia School of Medicine P.O. Box 800386 Charlottesville, VA 22908 E-mail: deboer@virginia.edu 